University of Warwick institutional repository: http://go.warwick.ac.uk/wrap This paper is made available online in accordance with publisher policies. Please scroll down to view the document itself. Please refer to the repository record for this item and our policy information available from the repository home page for further information.

To see the final version of this paper please visit the publisher's website. Access to the published version may require a subscription.

Author(s): X. Jian, S. Dixon, I. Baillie, R. S. Edwards, J. Morrison, and Y. Fan

Article Title: Shear wave generation using a spiral electromagnetic acoustic transducer

Year of publication: 2006

Link to published version: http://dx.doi.org/10.1063/1.2408644

Publisher statement: None 


\title{
Shear wave generation using a spiral electromagnetic acoustic transducer
}

\author{
X. Jian, ${ }^{\text {a) }}$ S. Dixon, I. Baillie, R. S. Edwards, J. Morrison, and Y. Fan \\ Department of Physics, University of Warwick, Coventry CV4 7AL, United Kingdom
}

(Received 28 July 2006; accepted 15 November 2006; published online 15 December 2006)

\begin{abstract}
A spiral electromagnetic acoustic transducer (EMAT) is efficient in eddy current generation and has been used for surface defect inspection using Rayleigh waves or thickness gauging based on plane waves in echo mode. Measured and calculated particle velocities and directivities are presented. It is found that the shear wave is not predominantly a plane wave. It has zero amplitude on the axis of the generation EMAT and has maximum amplitude at the critical angle. The shear wave could be used in the steel industry for both internal and surface defect inspections together with Rayleigh wave. (C) 2006 American Institute of Physics. [DOI: 10.1063/1.2408644]
\end{abstract}

Electromagnetic acoustic transducers (EMATs) are noncontact transducers that allow fast moving inspection in a hostile environment without disturbing the field being measured. ${ }^{1-9}$ EMAT ultrasonic generation and detection have been investigated by simulation and experimental measurement. ${ }^{1-9}$ Spiral EMATs, which are efficient in eddy current induction, have been used for surface defect inspection and thickness gauging. ${ }^{7}$ It is very interesting that both internal defects and surface defects can be inspected at the same time using a spiral EMAT. For this purpose, the characteristics of the Rayleigh wave and the bulk waves generated by a spiral EMAT must be fully investigated and optimized.

Optimal EMAT design and Rayleigh wave generation by a spiral EMAT have been well investigated. ${ }^{2,9}$ However, the spatial distribution of the shear wave generated by a spiral EMAT is still not clear. A spiral EMAT has been used for thickness gauging based on the round travel time of a plane shear wave. ${ }^{7}$ Does a spiral EMAT generate predominantly plane waves like a normal piston by means of a wedge?

A spiral EMAT constructed by a permanent magnet and a flat, uniformly hand-wound spiral coil of a diameter of $15 \mathrm{~mm}$ and 12 turns is to be investigated as an example for shear wave generation in steel that has a density of $7800 \mathrm{~kg} / \mathrm{m}^{3}$. The longitudinal and shear wave propagation velocities are 5.9 and $3.2 \mathrm{~km} / \mathrm{s}$, respectively. We have used EMATs to detect in-plane and out-of-plane particle velocities, since the EMATs have small apertures and do not touch the steel. ${ }^{9}$ The signal-to-noise ratios are about $35 \mathrm{~dB}$ for an EMAT sensitive to predominantly in-plane particle velocities and about $25 \mathrm{~dB}$ for an EMAT sensitive to predominantly out-of-plane particle velocities. ${ }^{8}$ Therefore, an EMAT sensitive predominantly in-plane particle velocity is used.

Figure 1 shows the measurement setup for a steel billet sample of $110 \mathrm{~mm}$ wide, $90 \mathrm{~mm}$ high, and $800 \mathrm{~mm}$ long. The generation EMAT is fixed while the receiving EMAT to investigate the through ultrasonic field is moved along the opposite surface for the B-scan. Figure 2 shows the measured (a) and calculated (b) B-scan images of particle velocities in a steel billet that is $90 \mathrm{~mm}$ thick. The calculation is done by finite element method using PZFLEX, where free boundary condition is assumed for the surfaces of the steel billet. ${ }^{10}$ Edge-related longitudinal (EC), edge shear waves (ES),

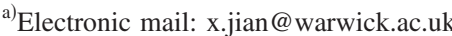

plane shear waves (PS), and head waves (HD) are all observed. The head wave is a shear wave arising at the sample surface due to the mode conversion of a surface skimming longitudinal wave. It has a straight-line wave front in the two-dimensional view, which is tangential to the wave front of the edge shear wave at one end and is connected to the surface skimming longitudinal wave front on the steel surface at the other end, propagating into the steel billet in the direction of the critical angle with the axis of the EMAT, which is given by $\theta_{\text {crt }}=\sin ^{-1}\left(v_{s} / v_{c}\right)$, where $v_{c}$ and $v_{s}$ are the propagation velocities of longitudinal and shear waves, respectively. For the steel billet discussed in this letter, $\theta_{\text {crt }}$ is $32.8^{\circ}$.

The direction of the in-plane component of the shear wave changes across the axis of the EMAT and the in-plane shear wave on the axis vanishes as shown in Fig. 2. The eddy current induced in the steel billet within interacts with the static magnetic field generating a radial-polarized Lorentz forced within the skin depth of steel billet. ${ }^{2}$ The Lorentz forces $F_{1}$ and $F_{2}$ at any two points $Q_{1}$ and $Q_{2}$ in Fig. 3, which are symmetrical to each other across the axis of the EMAT, are equal in amplitude and opposite in direction generating the shear waves $v_{1}$ and $v_{2}$, respectively. The in-plane components of $v_{1}$ and $v_{2}$ are equal in amplitude and opposite in direction, producing a total in-plane particle velocity of zero.

First-arrival wave arrival in Fig. 2 is the edge longitudinal wave, with an arrival time given by $t_{\mathrm{EC}}=\sqrt{x^{2}+y^{2}} / v_{c}$, where $y$ is the thickness of the sample, here $90 \mathrm{~mm}$. The

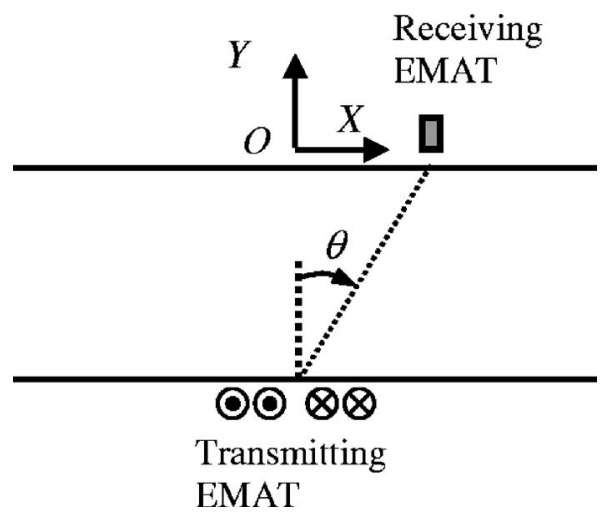

FIG. 1. B-scan measurement arrangement, showing the locations of each EMAT. 
(a)

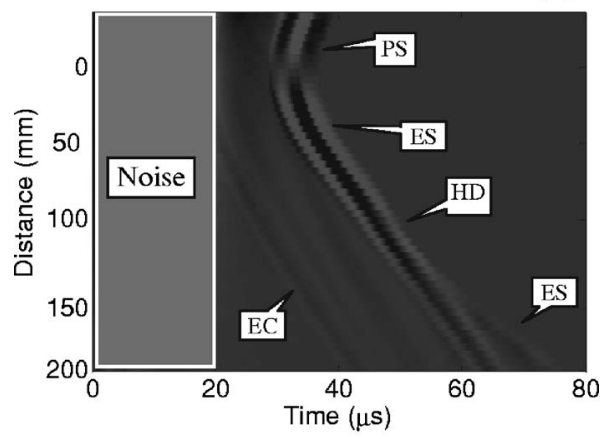

(b)

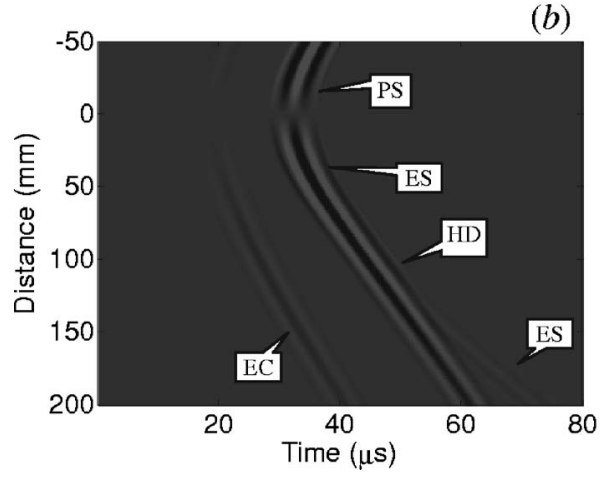

FIG. 2. Measured (a) and calculated (b) in-plane particle velocities on the opposite surface of the steel billet. The distance in the figure denotes the distance from the axis of the generation spiral EMAT.

arrival time of the plane shear wave, edge shear wave, and head wave within the above-mentioned zones are given, respectively, by $t_{\mathrm{PC}}=y / v_{s}, \quad t_{\mathrm{ES}}=\sqrt{x^{2}+y^{2}} / v_{s}, \quad t_{\mathrm{HD}}=(x$ $\left.+y / \tan \theta_{\text {crt }}\right) / v_{c}$.

The ratio of the arrival time of the edge longitudinal wave to that of first-arrival shear wave is not always $v_{s} / v_{c}$ as believed because the first-arrival shear is not always an edge shear wave or a plane shear wave but is related to $y$. As shown from Fig. 2, the first-arrival shear wave is a plane shear wave within the radius of the spiral coil, an edge shear wave outside the radius but within the critical angle, and a head wave beyond the critical angle. An observed separation of the head wave and the edge shear wave occurs at a dis-

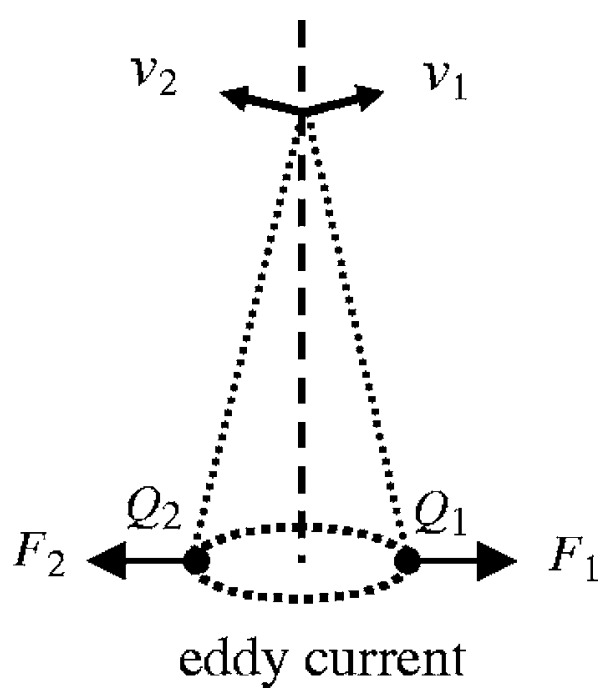

FIG. 3. Shear waves $\left(v_{1}\right.$ and $\left.v_{2}\right)$ on axis generated by the radial-polarized Lorentz force $\left(F_{1}\right.$ and $\left.F_{2}\right)$ of the spiral EMAT.

Lalc2 includes only head wave.
Downlo force $\left(F_{1}\right.$ and $\left.F_{2}\right)$ of the spiral EMAT.
01 Jul 2009 to 137.205 .202 .8 . Redistribution subject to AIP license or copyright; see http://apl.aip.org/apl/copyright.jsp

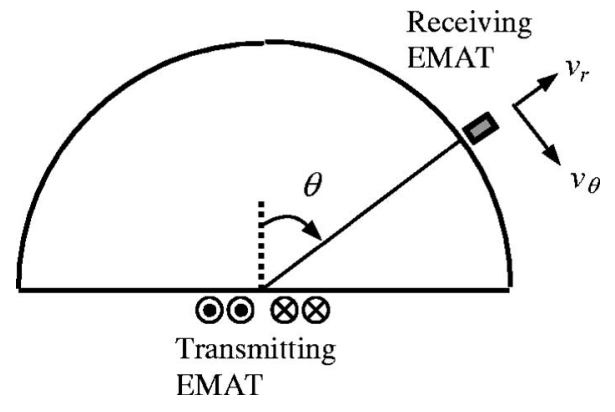

FIG. 4. Measurement setup for the directivity of a generation spiral EMAT.

tance of $130 \mathrm{~mm}$ in the $X$ axis in Fig. 2, corresponding to an angle of $\theta=55^{\circ}$ in the directivity shown in Fig. 2. The calculated separation should occur at a distance of $58 \mathrm{~mm}$ along the $X$ axis or at the critical angle of $32.8^{\circ}$ where the head wave is tangential to the edge shear wave. The discrepancy is due to the finite frequency bandwidth of the shear wave and the head wave causing overlapping, thereby preventing measurement of the difference of arrival times of head wave and edge shear wave, and due to the finite width of the receiving EMAT that acts to average the wave covered.

The in-plane and out-of-plane particle velocities of an edge shear wave are given, respectively, by $v_{\mathrm{ES} x}=v_{\mathrm{ES}} \cos \theta$ and $v_{\mathrm{ES} y}=v_{\mathrm{ES}} \sin \theta$, where $\theta$ is the angle with the axis of the generation EMAT. The in-plane and out-of-plane particle velocities of the head wave are given, respectively, by $v_{\mathrm{HD} x}$ $=v_{\mathrm{HD}} \cos \theta_{\mathrm{crt}}$ and $v_{\mathrm{HD} y}=v_{\mathrm{HD}} \sin \theta_{\mathrm{crt}}$.

The ratio of the in-plane to out-of-plane components of the edge wave is related to that of the head wave and is given by $v_{\mathrm{ES} x} / v_{\mathrm{ES} y}=\left(\tan \theta_{\mathrm{crt}} / \tan \theta\right)\left(v_{\mathrm{HD} x} / v_{\mathrm{HD} y}\right)$. At a position where $\theta$ is larger than the critical angle, the ratio of in-plane to out-of-plane components of the head wave is larger than that of the edge shear wave. Measurements based on a sensor with in-plane sensitivity therefore give a relatively stronger signal for the head wave than for the edge shear wave as shown in Fig. 1.

Figure 4 shows the typical measurement setup for transducer directivity. ${ }^{8}$ Figure 5 shows the calculated and measured directivities of the shear waves, calc1 and calc2. ${ }^{8}$ The calculated in-plane and out-of-plane velocities in Cartesian coordinate can be transformed into cylindrical coordinates by $v_{\theta}=v_{x} \cos \theta-v_{y} \sin \theta$ and $v_{r}=v_{x} \sin \theta+v_{y} \cos \theta{ }^{8}$ It is found that calc2 agrees well with the measured. Due to the destructive and constructive interference of the head wave and the edge shear wave, calc1 has several local minima and maxima.

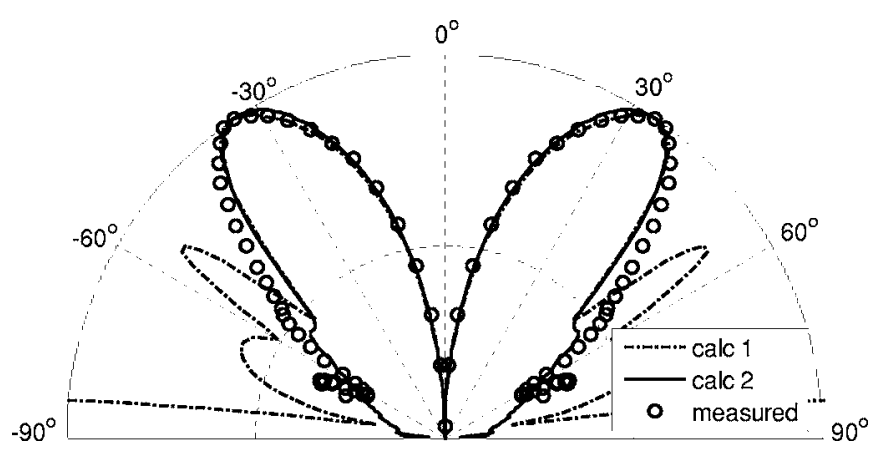

FIG. 5. Simulated (calc1 and calc2) and measured directivities of the generation spiral EMAT. calc1 includes both edge wave and head wave while AIP license or copyright; see http://apl.aip.org/apl/copyright.jsp 
In conclusion, we have studied the shear wave generation using a spiral EMAT. Measured and calculated particle velocities and directivities are presented. Unlike a piezoelectric transducer that generates predominantly plane shear waves by means of a wedge, a spiral EMAT generates shear waves that are not predominantly plane wave. First-arrival shear wave can be a plane, an edge, or a head wave dependent on the relative position of the detector to the generation EMAT. The in-plane component of the shear wave has zero amplitude on the axis of the generation EMAT due to the antisymmetry of the radial-polarized Lorentz force across the axis of the spiral EMAT, and the shear wave has maximum amplitude at the critical angle due to the constructive interference of the edge shear wave and head wave. For the detection of the shear wave in through transmission mode, a receiving EMAT is better situated on the opposite surface to the generation EMAT at the critical angle with the axis of the generation spiral EMAT.

${ }^{1}$ X. Jian, S. Dixon, R. S. Edwards, and J. Reed, J. Acoust. Soc. Am. 119, 2693 (2006).

${ }^{2}$ X. Jian, S. Dixon, K. Grattan, and R. Edwards, Sens. Actuators, A 128, 296 (2006).

${ }^{3}$ R. B. Thompson, J. Appl. Phys. 48, 4942 (1977).

${ }^{4}$ K. Kawashima, IEEE Trans. Sonics Ultrason. 31, 83 (1984).

${ }^{5}$ C. V. Dodd and W. E. Deeds, J. Appl. Phys. 39, 2829 (1968).

${ }^{6}$ H. Ogi, J. Appl. Phys. 82, 3940 (1997).

${ }^{7}$ S. Dixon., C. Edwards, and S. B. Palmer, Ultrasonics 39, 445 (2000).

${ }^{8}$ M. C. M. Bakker and M. D. Verweij, J. Acoust. Soc. Am. 113, 1850 (2003).

${ }^{9}$ X. Jian, S. Dixon, and S. B. Palmer, 2005 IEEE International Ultrasonics Symposium, Rotterdam, The Netherlands, 18-21 September 2005 (IEEE, New York, 2005), Vol. 2, p. 1276.

${ }^{10}$ X. Jian, Y. Fan, R. S. Edwards, and S. Dixon, J. Appl. Phys. 100, 064907 (2006). 